\title{
Spin detection in quantum dots by electric currents
}

\author{
Eugene G. Mishchenko, ${ }^{1,2}$ Arne Brataas, ${ }^{3}$ and Yaroslav Tserkovnyak ${ }^{1}$ \\ ${ }^{1}$ Lyman Laboratory of Physics, Harvard University, MA 02138, USA \\ ${ }^{2}$ L.D. Landau Institute for Theoretical Physics, Moscow 117334, Russia \\ ${ }^{3}$ Department of Physics, Norwegian University of Science and Technology, N-7491 Trondheim, Norway
}

\begin{abstract}
We develop a theoretical description of transport through quantum dots connected to reservoirs via spin-polarized ballistic contacts. Rate equations account for spin accumulation inside the dot, electron-electron interactions, and stochastic fluctuations. It is shown that both the ac response (admittance) and the frequency-dependent shot noise are governed by spin-flip scattering, which can be used to detect spin polarization and spin-flip processes in the dot.
\end{abstract}

PACS numbers: 73.63.Kv, 72.25.-b, 72.70.+m, 75.47.De

The discovery of the giant-magnetoresistance effect initiated a large activity aimed at understanding the transport properties of nanoscale devices involving ferromagnetic elements ${ }^{1}$. In such systems, transport is governed not only by the charge flow, but also by the spin flow, spin precession, and spin relaxation. The spin degree of freedom can provide new functionality in electronic devices and is envisaged as a future route of information processing. Until recently, the experimental and theoretical efforts have been mostly devoted to the exploration of the average current vs voltage-bias characteristics at a given static magnetic configuration. Recent fascinating developments concern spin-current-induced magnetization dynamics ${ }^{2}$. However, time-dependent transport properties in circuits with static magnetic elements have so far attracted little attention.

Current fluctuations are significant in submicron and nanoscale devices and provide additional information about correlation effects in such devices than the average conductance ${ }^{3.4}$. However, the information obtained from the second moment of current fluctuations (noise power) is often reduced by its universality, i.e., its independence of the particular realization of the device. There are (at least) two routes to achieve a further understanding beyond universality. The first option is to look for characteristics beyond a simple noise power, by elaborating the third (and higher) moments of current fluctuations. The second way is to search for mechanisms that break universality already for the second moment. In this Letter we follow the second route and report a way to study spin-dependent transport in quantum dots with spin-polarized contacts by measuring current fluctuations. The electron spin governs transport properties in spintronic and magnetoelectronic circuits. We show that noise properties depend on and can be used to study spin accumulation and spin-flip relaxation in quantum dots.

Surprisingly few studies exist on the noise in ferromagnet $(F)$ /normal-metal $(N)$ systems. Ref. 5 found superPoissonian noise in ferromagnetic single-electron transistors. Shot noise in the $F$ /quantum-dot $/ F$ system in the Coulomb-blockade regime was also considered in Refs. 6.78. The dependence of the noise on the relative magnetization orientation of the leads have been computed in double-barrier systems with tunnel, ballistic, or diffusive contacts9. Effects of a spin-flip scattering on the shot noise have been considered in Ref. 10 using the Boltzmann-Langevin approach for diffusive conductors connected to polarized leads. Its generalization to multiterminal systems has been given in Ref. 11. Ref. 12 studied similar effects using the Keldysh formalism assuming different chemical potentials for spin-up and spin-down electrons in the leads. The latter requires the system to be embedded into a larger non-equilibrium $F / N$ circuit which might have its own noise characteristics, though. Shot noise of spin-polarized entangled electrons has been considered in Ref. 13 in quantum dots and wires. Effects of Andreev reflection have recently been analyzed in Ref. 14 for an F-N-S system.

We study dc conductance, ac admittance and noise through quantum dots coupled via spin-polarized ballistic contacts. We go beyond the above-mentioned studies by computing the frequency-dependent admittance and the frequency-dependent noise, including electronelectron interactions. It is shown that the spin degree of freedom reveals itself both in the ac and dc properties. As we show below, the universality of the Fano factor is relaxed if the attached leads are spin polarized.

Quantum dots are spatially-confined conducting islands Ohmically contacted by metallic regions (reservoirs) and capacitively coupled by electrostatic gates. The electron motion inside the dot is usually chaotic as a result of the random scattering from boundaries and/or impurities. The size of the $\operatorname{dot} A$ enters its spectral and transport properties through the mean level spacing $\Delta=(\nu A)^{-1}$, which is inversely proportional to the density of states at the Fermi level, $\nu$. Throughout our discussion we assume the dot to be sufficiently large and open, for the effects of the Coulomb blockade to be unimportant. Nevertheless, Coulomb interactions are crucial and must be included via the capacitance of the dot. We consider a system where the central island of the dot is coupled to the left $(\mathrm{L})$ and right $(\mathrm{R})$ reservoirs via ballistic point contacts (PC's) supporting respectively $M_{L}$ and $M_{R}$ channels (per allowed spin direction).

Let us first review key results for the nonmagnetic systems. The theory for current fluctuations $\frac{15,16,17}{}$ is in 
a good agreement with experiment ${ }^{18}$. The average current $\bar{J}=V / R$ at voltage $V$ is related to the resistance $R=\left(h / 2 e^{2}\right)\left(M_{\mathrm{L}}^{-1}+M_{\mathrm{R}}^{-1}\right)$. (We disregard the quantum weak-localization corrections.) The fluctuations are typically characterized by the current-current correlation function

$$
S(\omega)=\int d t e^{i \omega\left(t-t^{\prime}\right)} \overline{\delta J(t) \delta J\left(t^{\prime}\right)},
$$

which for the dot at zero frequency and temperature is expressed via the Fano factor $F=M_{\mathrm{L}} M_{\mathrm{R}} / M^{2}(M=$ $\left.M_{\mathrm{L}}+M_{\mathrm{R}}\right)$ as $S(0)=e \bar{J} F$. The Fano factor, $F \leq 1$, relates fluctuations at zero temperature (i.e., the shot noise) to the average current and describes the suppression of the fluctuations below the Poissonian value $F=1$ characteristic for independent electron transmission (as, e.g., in tunnel junctions and vacuum diodes). For symmetric $\left(M_{L}=M_{R}\right)$ chaotic quantum dots, $F=1 / 4$, independent of its particular shape and realization.

We now turn to the study of a quantum dot with spinpolarized leads. We mainly focus on the most simple and illuminating case of two fully spin-polarized leads. In addition we also consider the case where only one the leads is spin polarized. We first present our results, before outlining the derivation.

Average dc current. We compute the average current through the quantum dot and find the resistance in the antiparallel configuration,

$$
R_{\mathrm{a}}=R_{\mathrm{L}}+R_{\mathrm{sf}}+R_{\mathrm{R}}
$$

where the resistances of the contacts are $R_{\mathrm{L}, \mathrm{R}}=$ $h /\left(e^{2} M_{\mathrm{L}, \mathrm{R}}\right)$ and the characteristic spin-flip resistance is $R_{\mathrm{sf}}=2 \Delta \tau_{s} / e^{2}$, in terms of the spin-flip relaxation time $\tau_{s} \frac{19}{}$. The resistance can be understood in terms of a circuit consisting of a series of the spin-polarized left and right $\mathrm{PC}$ resistances and a spin-flip resistance coupling two PC's 19 . Similarly, in the parallel configuration,

$$
R_{\mathrm{p}}=R_{\mathrm{L}}+R_{\mathrm{R}}
$$

The magnetoresistance of the dot is therefore determined by the spin-flip scattering rate: $R_{\mathrm{ap}}-R_{\mathrm{p}}=R_{\mathrm{sf}}$.

Admittance. General expressions for the frequency dependent admittance and noise in the arbitrary asymmetric geometry are lengthy, so we present below only the results for the symmetric $\left(M_{\mathrm{L}}=M_{\mathrm{R}}=M / 2\right)$ dot. The admittance is a $2 \times 2$ matrix, which for a symmetric dot is determined by just two quantities. Consider ac bias applied to the left lead, $V(t)=V_{\omega} e^{-i \omega t}$, while the right lead is kept at equilibrium. The current response $J_{L}(\omega)=G^{L L}(\omega) V_{\omega}, J_{R}(\omega)=G^{L R}(\omega) V_{\omega}$ is determined by the left, $G^{L L}$, and right, $G^{L R}$, admittance. In the parallel configuration the ac response is independent of the spin-flip relaxation,

$$
G_{\mathrm{p}}^{L L}=\frac{e^{2} M}{4 h} \frac{1-2 i \omega \tau_{C}}{1-i \omega \tau_{C}}, G_{\mathrm{p}}^{L R}=\frac{e^{2} M}{4 h} \frac{1}{1-i \omega \tau_{C}},
$$

where $\tau_{C}$ is the electrochemical $R C$ time of the dot

$$
\frac{1}{\tau_{C}}=\frac{1}{\tau_{d}}+\frac{e^{2} M}{h C},
$$

$C$ is the capacitance of the dot, and $\tau_{d}=h /(\Delta M)$ is the electron dwell time. In the antiparallel configuration,

$$
\left\{\begin{array}{l}
G_{\mathrm{a}}^{L L} \\
G_{\mathrm{a}}^{L R}
\end{array}\right\}=\left\{\begin{array}{l}
G_{\mathrm{p}}^{L L} \\
G_{\mathrm{p}}^{L R}
\end{array}\right\} \frac{\gamma-2 i \omega \tau_{d}}{1+\gamma-2 i \omega \tau_{d}}
$$

with $\gamma=2 \tau_{d} / \tau_{s}$ being the normalized spin-flip rate. For large spin-flip rates, $\gamma \rightarrow \infty$, equations (4) and (6) coincide and reproduce the result of Ref. 20. The admittance exhibits frequency dependence on the scale of $\tau_{d}^{-1}$, which in a typical experimental situation in GaAs quantum dots is much smaller than the charge relaxation rate, $\tau_{d}^{-1} \ll \tau_{C}^{-1}$. In the limit, $\omega \rightarrow 0$, the admittance (44) and (6) reduce to the static conductance, $G^{L L} \rightarrow G^{L R} \rightarrow R^{-1}$, as given by Eqs. (23).

Current fluctuations. The ac response reveals strong dependence on the polarization of the leads and the spinflip relaxation time, being governed by the degree of spin accumulation in the dot. Similar effects are revealed in the magnitude and spectrum of current fluctuations accompanying the dc current. Since the noise of a mesoscopic scatterer depends on its energy-relaxation processes, we make in the following a distinction between the elastic and inelastic transport regimes. We will restrict our attention to noise at small frequencies, $\hbar \omega \ll \max \left(e V, k_{B} T\right)$, and assume a typical situation when the charging energy exceeds the mean level spacing, $e^{2} / C \gg \Delta$.

Elastic transport through the dot. First we compute the Fano factor in the parallel configuration,

$$
F_{\mathrm{p}}^{\mathrm{e}}(\omega)=F_{\omega}+\frac{2\left(F_{\omega}-\frac{1}{4}\right)}{\gamma\left(1+\omega^{2} \tau_{s}^{2}\right)}, \quad F_{\omega}=\frac{1+2 \omega^{2} \tau_{C}^{2}}{4\left(1+\omega^{2} \tau_{C}^{2}\right)} .
$$

which in the typical scenario $\tau_{d}, \tau_{s} \gg \tau_{C}$ is very weakly dependent on the spin-flip scattering. Both for strong $\tau_{s} \rightarrow 0$ and weak $\tau_{s} \rightarrow \infty$ spin-flip scattering the expression (7) is reduced to the Fano factor for the unpolarized $\operatorname{dot} F_{\omega}$ exhibiting a crossover from the universal value of $F=1 / 4$ at low frequencies, $\omega \ll \tau_{C}^{-1}$, to $F=1 / 2$ at high frequencies, $\omega \gg \tau_{C}^{-1}$. To the best of our knowledge the expression for $F_{\omega}$ has never been explicitly presented before. [A general formalism has recently been presented for the noise in spin-independent mesoscopic conductors in Ref. 21 that, upon further elaboration, reproduces $F_{\omega}$ ].

The Fano factor in the antiparallel configuration differs significantly from $F_{\omega}$,

$$
F_{\mathrm{a}}^{\mathrm{e}}(\omega)=\frac{F_{\omega}(2+\gamma) \gamma^{2}\left(1+\omega^{2} \tau_{s}^{2}\right)+1+\gamma+\gamma^{2} / 2}{(1+\gamma)\left[(1+\gamma)^{2}+\gamma^{2} \omega^{2} \tau_{s}^{2}\right]},
$$

In the static regime, $\omega=0$, charging effects are irrelevant and the Fano factor reduces to

$$
F_{\mathrm{a}}^{\mathrm{e}}(0)=\frac{1+\gamma+\gamma^{2}+\gamma^{3} / 4}{(1+\gamma)^{3}}
$$


This formula describes a smooth crossover between Poissonian noise $F=1$ at $\gamma \rightarrow 0$ and a conventional value $F=1 / 4$ at $\gamma \rightarrow \infty$ characteristic for a spin-independent shot noise. The full Poissonian noise for weak spin-flip scattering is reminiscent of the shot noise in tunnel junctions. Indeed, current is suppressed at $\gamma \rightarrow 0$ and the transport through the dot is mostly due to the electrons that flip spin only once. Those electrons are few and therefore move independently inside the dot. Around $\omega \sim \tau_{s}^{-1}$ the Fano factor changes from the static value (9) to $(1+\gamma / 2) /[2(1+\gamma)]$ for $\tau_{s}^{-1} \ll \omega \ll \tau_{C}^{-1}$. With further increase of the frequency, the Fano factor rises up to $(1+\gamma / 2) /(1+\gamma)$ for $\omega \gg \tau_{C}^{-1}$. Experimentally, a situation with only one of the leads polarized might be of interest. The Fano factor for the zero-frequency noise in the elastic regime changes from $F=1 / 4$ for small spinflip, $\gamma \rightarrow 0$, to a slightly smaller value $F=2 / 9$ for large spin-flip, $\gamma \rightarrow \infty$.

The noise at finite temperatures in the elastic regime is calculated as well and expressed via the corresponding Fano factors in a usual way ${ }^{22}$. The situation is more interesting in the inelastic regime.

Inelastic transport through the dot. At elevated temperatures the (spin-conserving) electron-phonon scattering thermalizes the electronic distribution function. In the antiparallel configuration, the finite spin-flip relaxation prevents equilibration of electrons with opposite spin polarizations. The noise becomes

$$
\frac{S_{\mathrm{a}}^{\mathrm{i}}}{e \bar{J}}=\frac{2 k_{B} T \gamma}{e V(1+\gamma)}+\frac{1}{(1+\gamma)^{2}} \operatorname{coth}\left[\frac{e V}{2 k_{B} T(1+\gamma)}\right] .
$$

The second term in Eq. (10) is the nonequilibrium contribution due to the spin-flip scattering in the antiparallel configuration. The parallel configuration of the leads reveals the Nyquist-Johnson thermal noise only:

$$
S_{\mathrm{p}}^{\mathrm{i}} /(e \bar{J})=2 k_{B} T / e V .
$$

which vanishes in the low-temperature limit, $k_{B} T \ll e V$.

Derivation. Let us now outline the derivation of our results. The spin dynamics in the dot is determined by the rate equations for the deviations of the numbers of electrons $N_{\uparrow}$ and $N_{\downarrow}$ from their equilibrium values [assuming $\left.\hbar \omega \ll \max \left(e V, k_{B} T\right)\right]$ :

$$
\begin{aligned}
\frac{\partial N_{\uparrow}}{\partial t} & =J_{L} / e-\frac{N_{\uparrow}-N_{\downarrow}}{2 \tau_{s}}+\mathcal{L}(t), \\
\frac{\partial N_{\downarrow}}{\partial t} & =J_{R} / e+\frac{N_{\uparrow}-N_{\downarrow}}{2 \tau_{s}}-\mathcal{L}(t),
\end{aligned}
$$

here $J_{L}$ and $J_{R}$ are the electric currents entering the dot through the left and right PC's, respectively, and $\mathcal{L}(t)$ is a Langevin source due to the randomness of the spin-flip events. The current through the left quantum PC is

$$
\frac{J_{L}}{e}=\frac{M_{L}}{h}\left(\int d \epsilon\left[n_{L}-n_{0}\right]-N_{\uparrow} \Delta-\frac{e Q}{C}\right)+\mathcal{I}_{L}(t),
$$

where $Q$ denotes the charge imbalance in the dot: $\partial Q / \partial t=J_{L}+J_{R}$. A similar relation exists for the current through the right contact. The first two terms in the parentheses of Eq. (13) represent simply the current through the ballistic constriction, $n_{L}$ is the distribution function in the left lead and $n_{0}(\epsilon)$ is its equilibrium value. The third term describes the charging effect. Electrons experience the charge imbalance $Q$ in the dot as an effective change of the chemical potential $e Q / C$ (same for spin-up and spin-down electrons) of the central island having a capacitance $C$. The last term $\mathcal{I}_{L}(t)$ is the Langevin contribution describing intrinsic stochastic noise in the PC. This term vanishes on average and has the correlator with the white-noise spectrum

$$
\begin{aligned}
\overline{\mathcal{I}_{L}(t) \mathcal{I}_{L}\left(t^{\prime}\right)}= & \delta\left(t-t^{\prime}\right) \frac{M_{L}}{h} \\
& \times \int d \epsilon\left[n_{L}\left(1-n_{L}\right)+\bar{f}_{\uparrow}\left(1-\bar{f}_{\uparrow}\right)\right],(14)
\end{aligned}
$$

and similarly for the right quantum PC, but with $\bar{f}_{\downarrow}$ on the right-hand side. The mean (averaged over fluctuations) distribution of electrons inside the $\operatorname{dot}, \bar{f}_{\uparrow}(\epsilon)$, $\bar{f}_{\downarrow}(\epsilon)$, is homogeneous and isotropic (as a result of the multiple chaotic scattering). The Langevin term $\mathcal{L}(t)$ in Eq. (12) describes the randomness of the spin-flip relaxation 10 :

$$
\left.\overline{\mathcal{L}(t) \mathcal{L}\left(t^{\prime}\right)}=\frac{\delta\left(t-t^{\prime}\right)}{2 \Delta \tau_{s}} \int d \epsilon \overline{f_{\uparrow}}\left(1-\bar{f}_{\downarrow}\right)+\bar{f}_{\downarrow}\left(1-\bar{f}_{\uparrow}\right)\right]
$$

This expression implies that separate events of spinflip relaxation are independent and therefore Poissonian. They are also assumed to be elastic and have a constant scattering rate around the Fermi energy.

Elastic transport through the dot. When the inelastic (e.g., electron-phonon) scattering time exceeds the dwell time in the dot, the energy of an electron is conserved during its transport across the dot. The mean electron distribution function is then found from the condition that the incoming flow of spin-up electrons through the left $\mathrm{PC}$ equals the total rate of spin-flip transition (since there are no spin-up electrons leaving the dot via the right quantum $\mathrm{PC}$ ),

$$
\frac{M_{L} \Delta}{h}\left[n_{L}(\epsilon)-\bar{f}_{\uparrow}(\epsilon)\right]=\frac{\bar{f}_{\uparrow}(\epsilon)-\bar{f}_{\downarrow}(\epsilon)}{2 \tau_{s}} .
$$

with a similar condition holding for spin-down electrons in the right PC. Combining the two relations, we obtain (omitting the energy dependence)

$$
\left\{\begin{array}{c}
\bar{f}_{\uparrow} \\
\bar{f}_{\downarrow}
\end{array}\right\}=\frac{\left\{\begin{array}{c}
n_{L} \\
n_{R}
\end{array}\right\}+\gamma \bar{f}}{1+\gamma}, \quad \bar{f}=\frac{n_{L} M_{L}+n_{R} M_{R}}{M_{L}+M_{R}} .
$$

For large spin-flip scattering rate, $\gamma \gg 1$, the distribution function becomes spin independent and equal to $\bar{f}$.

Let us calculate the average current through the dot under dc bias, $n_{L}(\epsilon)=n_{0}(\epsilon-e V), n_{R}(\epsilon)=n_{0}(\epsilon)$. In a 
steady state the dot is electrically neutral, $Q=0$. Substituting $\bar{N}_{\uparrow}=\Delta^{-1} \int d \epsilon\left[\bar{f}_{\uparrow}(\epsilon)-n_{0}(\epsilon)\right]$, we obtain the current through the $\operatorname{dot} \bar{J}=V / R$ with the resistance in the parallel and antiparallel configurations given by equations (2) and (3) respectively.

The stochastic Langevin sources $\mathcal{I}$ and $\mathcal{L}$ describe the fluctuations around the mean solution, Eq. (17). The charge imbalance is given by $Q=e\left(\delta N_{\uparrow}+\delta N_{\downarrow}\right)$, with the fluctuating quantities $\delta N_{\uparrow}, \delta N_{\downarrow}$ determined from the solution of the coupled inhomogeneous equations (12) around the steady state. The fluctuation of the electric current is then found from Eq. (13). The calculations are rather straightforward in the Fourier representation. E.g. in the case of a symmetric $\operatorname{dot}\left(M_{L}=M_{R}\right)$ with charging energy exceeding the mean level spacing $\left(e^{2} / C \gg \Delta\right)$ and the antiparallel orientation of the leads, we find,

$$
\frac{\delta J_{L}}{e}=\frac{\gamma\left(1-i \omega \tau_{s}\right)\left[\left(\frac{1}{2}-i \omega \tau_{C}\right) \mathcal{I}_{L}-\mathcal{I}_{R}\right]-\left(1-i \omega \tau_{C}\right) \mathcal{L}}{\left(1-i \omega \tau_{C}\right)\left(1+\gamma-i \gamma \omega \tau_{s}\right)}
$$

The calculation of the noise power is now performed with the help of the correlators (14) and (15) giving the frequency-dependent Fano factors in (7) and (8).

Inelastic transport through the dot. When the temperature $T$ increases, the inelastic scattering changes dramatically the noise pattern. Once the characteristic electron-phonon scattering time drops below the dwell time in the dot, the electron distribution $\bar{f}_{\alpha}(\epsilon)$ relaxes to the Fermi-Dirac distribution. The chemical potentials of spin-up and spin-down electrons are found from the current-conservation conditions for the right and left PC's. For the antiparallel configuration: $\mu_{\uparrow}=e V\left(M+\gamma M_{L}\right) /[(1+\gamma) M], \mu_{\downarrow}=e V \gamma M_{L} /[(1+\gamma) M]$. It can be easily verified that the resistance in the inelastic regime is still given by Eq. (2). The above expression (18) for the fluctuation of electric current holds in this case as well. Calculating the static noise power in a symmetric dot at finite temperature with the help of correlators (14) and (15), we find expressions (10) and (11).

In conclusion, we have presented a framework for the computation of spin-dependent transport in quantum dots based on the Langevin rate equations. Its application is illustrated by finding the ac admittance and the frequency-dependent noise in dots coupled with reservoirs via spin-polarized ballistic contacts. Timedependent measurements seem to be a much more powerful tool for studying spintronic effects than dc measurements. In particular, the knowledge of the dc magnetoresistance determines only the ratio of the spin-flip scattering rate $\tau_{s}^{-1}$ to the mean level spacing $\Delta$. Moreover, when $\tau_{s}^{-1} \gg M \Delta$ the magnetoresistance represents only a small fraction of the total resistance, making the former difficult to extract. On the other hand, timedependent measurements of the ac admittance and noise spectrum should reveal $\tau_{s}$ without any restrictions imposed by the magnitude of the mean level spacing and the number of channels coupled to the dot.

We are grateful to B. I. Halperin for useful discussions and his great hospitality during A.B.'s stay at Harvard University where most of this work was carried out. Communications with Ya. M. Blanter are also acknowledged. The work was supported by the NSF Grant PHY-0117795, DARPA Award No. MDA 972-01-1-0024, and by the Harvard Society of Fellows.
1 M. A. M. Gijs and G. E. W. Bauer, Adv. Phys. 46, 285 (1997).

2 J. C. Slonczewski, J. Magn. Magn. Mater. 159, L1 (1996); L. Berger, Phys. Rev. B 54, 9353 (1996); Y. Tserkovnyak, A. Brataas, and G. E. W. Bauer, Phys. Rev. B 67, 140404(R) (2003), and references therein.

3 C. W. J. Beenakker and C. Schönenberger, Phys. Today, 56, 37 (2003).

4 Ya. M. Blanter and M. Büttiker, Phys. Rep. 336, 1 (2000).

5 B. R. Bulka et al., Phys. Rev. B 60, 12246 (1999).

${ }^{6}$ R. Lu, Z.-R. Liu, cond-mat/0210350 (unpublished).

7 F. M. Souza, J. C. Egues, A.-P. Jauho, cond-mat/0209263 (unpublished).

8 R. Lopez and D. Sanchez, Phys. Rev. Lett. 90, 116602 (2003).

9 Y. Tserkovnyak and A. Brataas, Phys. Rev. B 64, 214402 (2001).

10 E. G. Mishchenko, Phys. Rev. B 68, 100409(R) (2003).

11 W. Belzig and M. Zareyan, cond-mat/0307070 (unpublished); A. Cottet, W. Belzig, and C. Bruder, cond-mat/0308564 (unpublished).

12 A. Lamacraft, cond-mat/0304544 (unpublished).

13 J. C. Egues, G. Burkard and D. Loss, Phys. Rev. Lett. 89, 176401 (2002); J. C. Egues et al., in Quantum Noise in Mesoscopic Physics, NATO Science Series 97, 241-274, (Kluwer, Dordrecht, 2003).

14 D. Sanchez, R. Lopez, P. Samuelsson, and M. Buttiker, cond-mat/0306132 (unpublished).

15 R. A. Jalabert, J.-L. Pichard, and C. W. J. Beenakker, Europhys. Lett. 27, 255 (1994).

16 Yu. V. Nazarov, in Quantum Dynamics of Submicron Structures, edited by H. A. Cerdeira, B. Kramer, and G. Schön, NATO ASI Series E291 (Kluwer, Dordrecht), p. 687 (1995).

17 Ya. M. Blanter and E. V. Sukhorukov, Phys. Rev. Lett. 84, 1280 (2000).

18 S. Oberholzer et al., Phys. Rev. Lett. 86, 2114 (2001).

19 A. Brataas, Yu. V. Nazarov, J. Inoue, and G. E. W. Bauer, Phys. Rev. B 59, 93 (1999); Eur. Phys. J. B 9, 421 (1999).

20 P. Brouwer and M. Büttiker, Europhys. Lett. 37, 441 (1997).

21 K. E. Nagaev, S. Pilgram, and M. Büttiker, cond-mat/0306465 (unpublished).

22 The relation between the Fano factor and the static noise power for arbitrary ratio of the voltage and temperature, $S^{\mathrm{e}} /(e \bar{J})=2 k_{B} T\left(1-F^{\mathrm{e}}\right) / e V+F^{\mathrm{e}} \operatorname{coth}\left(e V / 2 k_{B} T\right)$. See, e.g. Ref. 4 . 